\title{
Vortex Identification in the Wall Region of Turbulent Channel Flow
}

\author{
Giancarlo Alfonsi $i^{1}$ and Leonardo Primavera ${ }^{2}$ \\ ${ }^{1}$ Dipartimento di Difesa del Suolo, Università della Calabria \\ Via P. Bucci 42b, 87036 Rende (Cosenza), Italy \\ alfonsi@dds.unical.it \\ ${ }^{2}$ Dipartimento di Fisica, Università della Calabria \\ Via P. Bucci 33b, 87036 Rende (Cosenza), Italy \\ lprimavera@fis.unical.it
}

\begin{abstract}
Four widely-used techniques for vortex detection in turbulent flows are investigated and compared. The flow of a viscous incompressible fluid in a plane channel is simulated numerically by means of a parallel computational code based on a mixed spectral-finite difference algorithm for the numerical integration of the Navier-Stokes equations. The DNS approach (Direct Numerical Simulation) is followed in the calculations, performed at friction Reynolds number $R e_{\tau}=180$. A database representing the turbulent statistically steady state of the velocity field through 10 viscous time units is assembled and the different vortex-identification techniques are applied to the database. It is shown that the method of the "imaginary part of the complex eigenvalue pair of the velocity-gradient tensor" gives the best results in identifying hairpin-like vortical structures in the wall region of turbulent channel flow.
\end{abstract}

Keywords: turbulence, direct numerical simulation, wall-bounded flows, vortex-eduction methods.

\section{Introduction}

Organized vortical structures in wall-bounded flows have been investigated by several authors. One of the first contributions to the issue of the presence of vortices in the wall region of turbulent shear flows is due to Theodorsen [1] who introduced the hairpin vortex model. Robinson [2] confirmed the existence of non-symmetric archand quasi-streamwise vortices on the basis of the evaluation of DNS results. Studies involving the dynamics of hairpin vortices in the boundary layer have been performed by Perry \& Chong [3], Acarlar \& Smith [4,5], Smith et al. [6], Haidari \& Smith [7] and Singer \& Joslin [8]. On these bases, a picture of vortex generation and reciprocal interaction in the boundary layer emerges in which processes of interaction of existing vortices with wall-layer fluid involve viscous-inviscid interaction, generation of new vorticity, redistribution of existing vorticity, vortex stretching near the wall and vortex relaxation in the outer region. The process of evolution of a hairpin vortex involves the development of vortex legs in regions of increasing shear with intensification of vorticity in the legs themselves. The leg of a vortex - considered in isolation - may 
appear as a quasi-streamwise vortex near the wall. The head of a vortex instead, rises through the shear flow, entering regions of decreasing shear. As a consequence, the vorticity in the vortex head diminishes.

In spite of the remarkable amount of scientific work accomplished in this field, still there are no definite conclusions on the character of the phenomena occurring in the wall region of wall-bounded turbulent flows. Modern techniques for the numerical integration of the Navier-Stokes equations (advanced numerical methods and highperformance computing) have the ability of remarkably increasing the amount of data gathered during a research of computational nature, bringing to the condition of managing large amounts of data. A typical turbulent-flow database includes all three components of the fluid velocity in all points of a three-dimensional domain, evaluated for an adequate number of time steps of the turbulent statistically steady state. Mathematically-founded methods for the identification of vortical structures from a turbulent-flow database have been introduced by: i) Perry \& Chong [9], based on the complex eigenvalues of the velocity-gradient tensor; ii) Hunt et al. [10] and Zhong et al. [11], based on the second invariant of the velocity-gradient tensor; iii) Zhou et al. [12], based on the imaginary part of the complex eigenvalue pair of the velocity-gradient tensor; iv) Jeong \& Hussain [13], based on the analysis of the Hessian of the pressure. These techniques for vortex eduction are extensively used in turbulence research but no work exists in which their ability in vortex detection is systematically compared. In the present work the capability of vortex identification of the four techniques outlined above, is analyzed.

\section{Vortex-Identification Techniques}

\subsection{Complex Eigenvalues of the Velocity-Gradient Tensor $(\operatorname{Method} A)$}

Perry \& Chong [9] proposed a definition of a vortex as a region of space where the rate-of-deformation tensor has complex eigenvalues. By considering the system of the Navier-Stokes equations, an arbitrary point $O$ can be chosen in the flow field and a Taylor series expansion of each velocity component can be performed in terms of the space coordinates, with the origin in $O$. The first-order pointwise linear approximation at point $O$ is:

$$
u_{i}=\dot{x}_{i}=A_{i}+A_{i j} x_{j}
$$

and if $O$ is located at a critical point, the zero-order terms $A_{i}$ are equal to zero, being $A_{i j}=\partial u_{i} / \partial x_{j}$ the velocity-gradient tensor (rate-of-deformation tensor, $\boldsymbol{A}=\nabla \boldsymbol{u}$ ). In the case of incompressible flow, the characteristic equation of $A_{i j}$ becomes:

$$
\lambda^{3}+Q \lambda+R=0
$$

where $Q$ and $R$ are invariants of the velocity-gradient tensor (the other invariant $P=0$ by continuity). Complex eigenvalues of the velocity-gradient tensor occur when the discriminant of $A_{i j}, D>0$. According to this method, whether or not a 
region of vorticity appears as a vortex depends on its environment, i.e. on the local rate-of-strain field induced by the motions outside of the region of interest.

\subsection{Second Invariant of the Velocity-Gradient Tensor (Method B)}

Hunt et al. [10] and Zhong et al. [11] devised another criterion, in defining a eddy zone a region characterized by positive values of the second invariant $Q$ of the velocity-gradient tensor. The velocity-gradient tensor can be split into symmetric and antisymmetric parts:

$$
A_{i j}=S_{i j}+W_{i j}
$$

being $S_{i j}$ the rate-of-strain tensor (corresponding to the pure irrotational motion) and $W_{i j}$ the rate-of-rotation tensor (corresponding to the pure rotational motion). The second invariant of $A_{i j}$ can be written as:

$$
Q=\left(W_{i j} W_{i j}-S_{i j} S_{i j}\right) / 2
$$

where the first term on the right-hand side of (4) is proportional to the enstrophy density and the second term is proportional to the rate of dissipation of kinetic energy.

\subsection{Imaginary Part of the Complex Eigenvalue Pair of the Velocity-Gradient Tensor (Method C)}

Zhou et al. [12] adopted the criterion of identifying vortices by visualizing isosurfaces of the imaginary part of the complex eigenvalue pair of the velocity-gradient tensor (actually the square of). By considering equation (2) and defining the quantities:

$$
J=\left(-\frac{R}{2}+\sqrt{\frac{R^{2}}{4}+\frac{Q^{3}}{27}}\right)^{\frac{1}{3}}, \quad K=-\left(+\frac{R}{2}+\sqrt{\frac{R^{2}}{4}+\frac{Q^{3}}{27}}\right)^{\frac{1}{3}}
$$

one has:

$$
\lambda_{1}=J+K, \quad \lambda_{2}=-\frac{J+K}{2}+\frac{J-K}{2} \sqrt{-3}, \quad \lambda_{3}=-\frac{J+K}{2}-\frac{J-K}{2} \sqrt{-3} .
$$

The method of visualizing isosurfaces (of the square) of the imaginary part of the complex eigenvalue pair of the velocity-gradient tensor is frame independent and due to the fact that the eigenvalue is complex only in regions of local circular or spiralling streamline, it automatically eliminates regions having vorticity but no local spiralling motion, such as shear layers.

\subsection{Analysis of the Hessian of the Pressure (Method D)}

Jeong \& Hussain [13] proposed a definition of a vortex by reasoning on the issue of the pressure minimum, as follows “... a vortex core is a connected region characterized by two negative eigenvalues of the tensor $\boldsymbol{B}=\boldsymbol{S}^{2}+\boldsymbol{W}^{2} \ldots$..", where $\boldsymbol{S}$ 
and $\boldsymbol{W}$ are the symmetric and antisymmetric parts of the velocity-gradient tensor. The gradient of the Navier-Stokes equation is considered and decomposed into symmetric and antisymmetric parts. By considering the symmetric part (the antisymmetric portion is the vorticity-transport equation), one has:

$$
\frac{D S_{i j}}{D t}-v \frac{\partial}{\partial x_{k} \partial x_{k}} S_{i j}+B_{i j}=-\frac{1}{\rho} \frac{\partial p}{\partial x_{i} \partial x_{j}}
$$

where:

$$
B_{i j}=S_{i k} S_{k j}+W_{i k} W_{k j} .
$$

The existence of a local pressure minimum requires two positive eigenvalues for the Hessian tensor $\left(\partial p / \partial x_{i} \partial x_{j}\right)$. By neglecting the contribution of the first two terms on the left-hand side of equation (7), only tensor (8) is considered to determine the existence of a local pressure minimum due to a vortical motion, i.e. the presence of two negative eigenvalues of $\boldsymbol{B}$. The tensor $\boldsymbol{B}$ is symmetric by construction, all its eigenvalues are real and can be ordered $\left(\lambda_{1} \geq \lambda_{2} \geq \lambda_{3}\right)$. According to this method a vortex is defined as a connected region of the flow with the requirement that the intermediate eigenvalue of $\boldsymbol{B}, \lambda_{2}<0$.

\section{Numerical Simulations}

The numerical simulations are performed with a parallel computational code based on a mixed spectral-finite difference technique. The unsteady Navier-Stokes equation (besides continuity) for incompressible fluids with constant properties in three dimensions and non-dimensional conservative form, is considered ( $i \& j=1,2,3)$ :

$$
\frac{\partial u_{i}}{\partial t}+\frac{\partial}{\partial x_{j}}\left(u_{i} u_{j}\right)=-\frac{\partial p}{\partial x_{i}}+\frac{1}{R e_{\tau}} \frac{\partial^{2} u_{i}}{\partial x_{j} \partial x_{j}}
$$

where $u_{i}(u, v, w)$ are the velocity components in the cartesian coordinate system $x_{i}(x, y, z)$. Equation (9) is nondimensionalized by the channel half-width $h$ for lenghts, wall shear velocity $u_{\tau}$ for velocities, $\rho u_{\tau}^{2}$ for pressure and $h / u_{\tau}$ for time, being $R e_{\tau}=\left(u_{\tau} h / v\right)$ the friction Reynolds number. The fields are admitted to be periodic in the streamwise $(x)$ and spanwise $(z)$ directions, and equation (9) are Fourier transformed accordingly. The nonlinear terms in the momentum equation are evaluated pseudospectrally by anti-transforming the velocities back in physical space to perform the products (FFTs are used). In order to have a better spatial resolution near the walls, a grid-stretching law of hyperbolic-tangent type is introduced for the grid points along $y$, the direction orthogonal to the walls. For time advancement, a third-order Runge-Kutta algorithm is implemented and time marching is executed with the fractional-step method. No-slip boundary conditions at the walls and cyclic conditions in the streamwise and spanwise directions are applied to the velocity. More detailed descriptions of the numerical scheme, of its reliability and of the performance obtained on the parallel computers that have been used, can be found in Alfonsi et al. 
[14] and Passoni et al. $[15,16,17]$. The characteristic parameters of the numerical simulations are the following. Computing domain: $L_{x}^{+}=1131, L_{y}^{+}=360, L_{z}^{+}=565$ (wall units). Computational grid: $N_{x}=96, N_{v}=129, N_{z}=64$. Grid spacing: $\Delta x^{+}=11.8, \Delta y_{\text {center }}^{+}=4.4, \Delta y_{\text {wall }}^{+}=0.87, \Delta z^{+}=8.8$ (wall units). It can be verified that there are 6 grid points in the $y$ direction within the viscous sublayer $\left(y^{+} \leq 5\right)$. After the insertion of appropriate initial conditions, the initial transient of the flow in the channel is simulated, the turbulent statistically steady state is reached and calculated for a time $t=10 \delta / u_{\tau}\left(t^{+}=1800\right)$. 20000 time steps are calculated with a temporal resolution of $\Delta t=5 \times 10^{-4} \delta / u_{\tau}\left(\Delta t^{+}=0.09\right)$. In Figure 1, the computed turbulence intensities (in wall units) of present work are compared with the results of Moser et al. [18] at $R e_{\tau}=180$. The agreement between the present results and the results of Moser et al. [18] (obtained with a fully spectral code) is rather satisfactory.

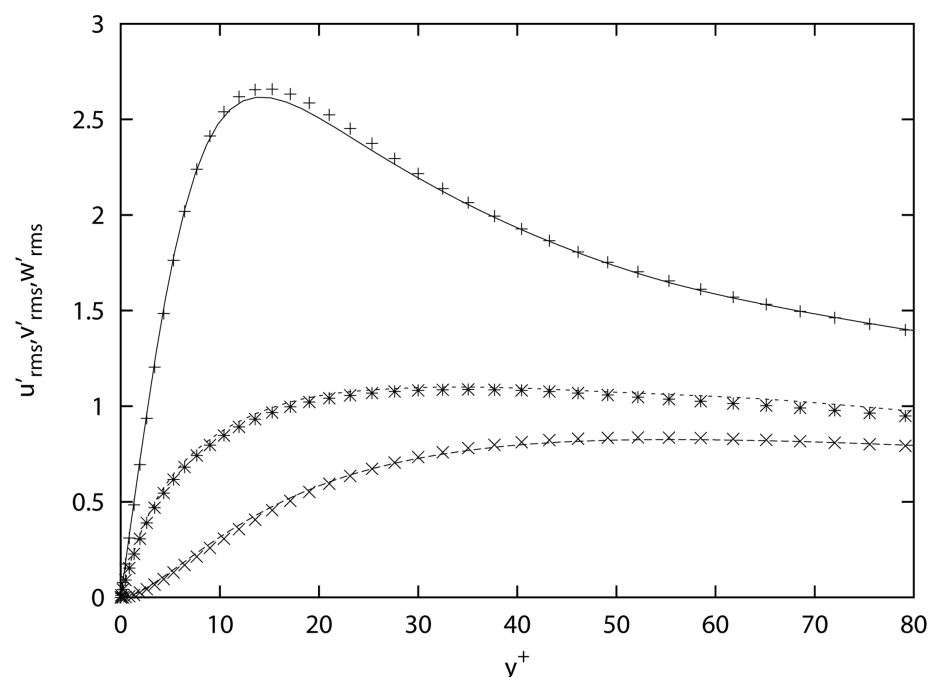

Fig. 1. Rms values of the velocity fluctuations normalized by the friction velocity in wall coordinates. Present work: $(-)\left(u_{r m s}^{\prime}\right),(--)\left(v_{r m s}^{\prime}\right),(\cdots)\left(w_{r m s}^{\prime}\right)$. Data from Moser et al. [18]: (+) $\left(u_{r m s}^{\prime}\right),(\mathbf{x})\left(v_{r m s}^{\prime}\right),(*)\left(w_{r m s}^{\prime}\right)$.

\section{Results}

In Figures $2 a-b$ the vortical structure that occurs at $t^{+}=1065.6$ as detected with method $A$, is represented and visualized from two different points of view (isosurfaces corresponding to the $5 \%$ of the maximum value are used in all representations). The top and side views show a structure not very well corresponding to a hairpin vortex. Portions of head and legs are visible, being the neck almost missing. The visualizations of the flow structure shows in practice a sketch of an hairpin, that can be completed only by intuition. Of the four methods examined, method $A$ gives the less satisfactory representation of the flow structure at the bottom wall of the computational domain at $t^{+}=1065.6$. 

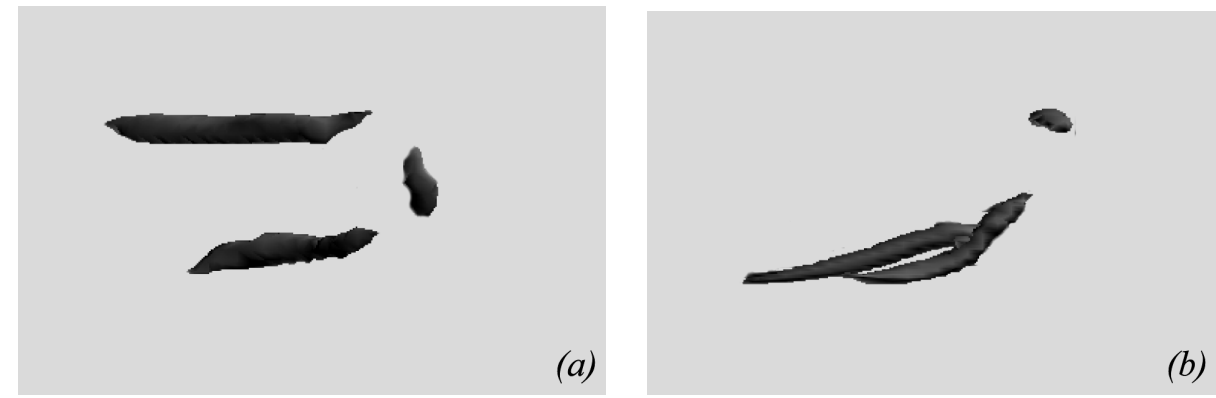

Fig. 2. Method A. Representation of hairpin vortex: $a$ ) top view; $b$ ) side view.
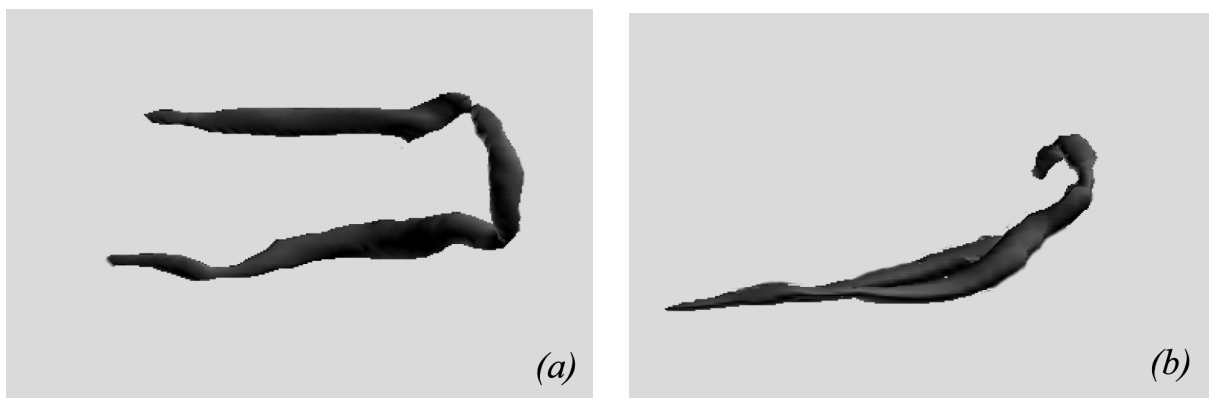

Fig. 3. Method $B$. Representation of hairpin vortex: $a$ ) top view; $b$ ) side view.
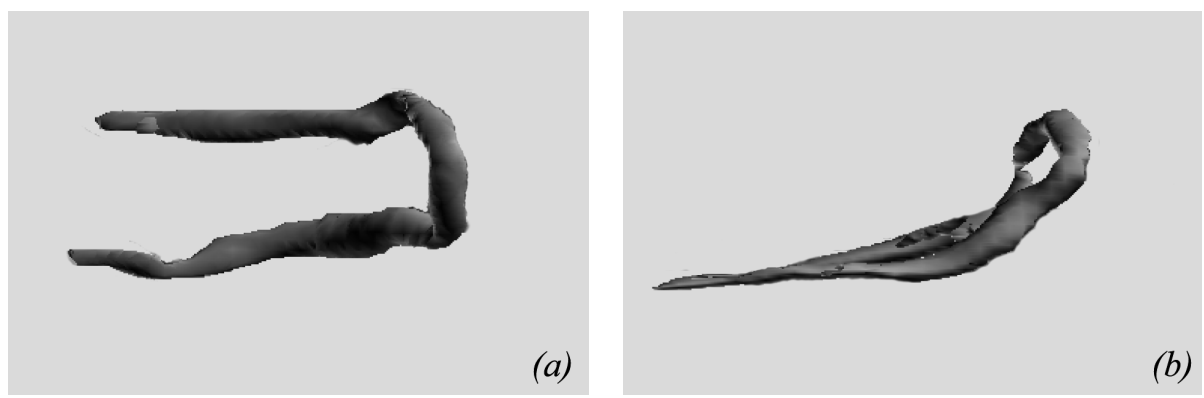

Fig. 4. Method $C$. Representation of hairpin vortex: $a$ ) top view; $b$ ) side view.

In Figures $3 a-b$ the flow structure at as educted with method $B$, is represented. A hairpin-like vortical structure more complete with respect to the former case is visible. The head of the vortex is almost complete and well defined. Of the two legs, one is longer than the other and both are longer with respect to those of method $A$. In turn, a portion of the vortex neck is missing, as can be seen from Figure $3 b$. Figures $4 a-b$ show the vortex structure extracted with method $C$. The Figures show a complete and well-defined hairpin vortex, with legs, neck and head clearly represented and no missing parts anywhere. Of the four eduction techniques tested, this is the best 

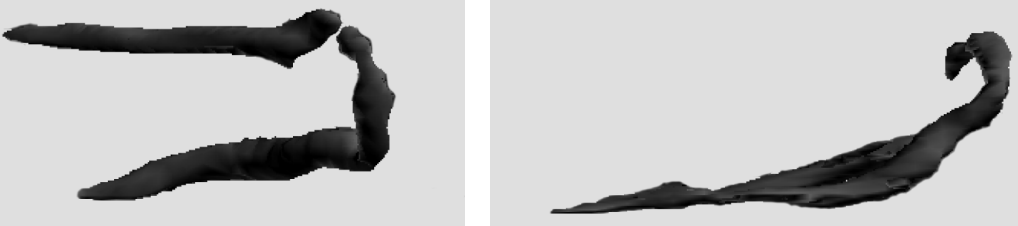

(a)

Fig. 5. Method $D$. Representation of hairpin vortex: $a$ ) top view; $b$ ) side view.

representation that can be obtained. From Figure $4 a$ it can be noted that the hairpin exhibits two legs of length comparable to those shown in Figure $3 a$. Figure $4 b$ shows a hairpin neck remarkably more thick with respect to that of Figure $3 b$. The results obtained with the use of method $D$ are shown in Figures $5 a-b$. Also in this case a not complete hairpin vortex structure appears. Figure $5 b$ shows that a portion of the neck of the vortex is missing.

\section{Concluding Remarks}

The case of the flow of a viscous incompressible fluid in a plane channel is simulated numerically at $R e_{\tau}=180$ and a turbulent-flow database is assembled. Four different criteria for vortex eduction are applied to the database and compared, showing that: $i$ ) the method of the "complex eigenvalues of the velocity-gradient tensor" gives the less satisfactory results in terms of vortex representation; ii) the methods of the "second invariant of the velocity-gradient tensor" and that based on the "analysis of the Hessian of the pressure" gives intermediate results, in the sense that a hairpin-like vortical structure actually appears, otherwise with missing parts or not optimal definitions; iii) the best results are obtained by using the method of the "imaginary part of the complex eigenvalue pair of the velocity-gradient tensor".

\section{References}

1. Theodorsen, T.: Mechanism of turbulence. In Proc. $2^{\text {nd }}$ Midwestern Mechanics Conf. (1952) 1

2. Robinson, S.K.: Coherent motions in the turbulent boundary layer. Annu. Rev. Fluid Mech. 23 (1991) 601

3. Perry, A.E., Chong, M.S.: On the mechanism of wall turbulence. J. Fluid Mech. 119 (1982) 173

4. Acarlar, M.S., Smith, C.R.: A study of hairpin vortices in a laminar boundary layer. Part 1. Hairpin vortices generated by a hemisphere protuberance. J. Fluid Mech. 175 (1987) 1

5. Acarlar, M.S., Smith, C.R.: A study of hairpin vortices in a laminar boundary layer. Part 2. Hairpin vortices generated by fluid injection. J. Fluid Mech. 175 (1987) 43 
6. Smith, C.R., Walker, J.D.A., Haidari A.H., Soburn U.: On the dynamics of near-wall turbulence. Phil. Trans. R. Soc. A 336 (1991) 131

7. Haidari, A.H., Smith, C.R.: The generation and regeneration of single hairpin vortices. $J$. Fluid Mech. 277 (1994) 135

8. Singer, B.A., Joslin R.D.: Metamorphosis of a hairpin vortex into a young turbulent spot. Phys. Fluids 6 (1994) 3724

9. Perry, A.E., Chong, M.S.: A description of eddying motions and flow patterns using critical-point concepts. Annu. Rev. Fluid Mech. 19 (1987) 125

10. Hunt, J.C.R., Wray, A.A., Moin, P.: Eddies, streams and convergence zones in turbulent flows. In Proc. Center Turbulence Research 1988 Summer Prog. NASA Ames/Stanford University (1988) 193

11. Zhong, J., Huang, T.S., Adrian, R.J.: Extracting 3D vortices in turbulent fluid flow. IEEE Trans. Patt. Anal. Machine Intell. 20 (1998) 193

12. Zhou, J., Adrian, R.J., Balachandar, S., Kendall, T.M.: Mechanisms for generating coherent packets of hairpin vortices in channel flow. J. Fluid Mech. 387 (1999) 353

13. Jeong, J., Hussain, F.: On the definition of a vortex. J. Fluid Mech. 285 (1995) 69

14. Alfonsi, G., Passoni, G., Pancaldo, L., Zampaglione D.: A spectral-finite difference solution of the Navier-Stokes equations in three dimensions. Int. J. Num. Meth. Fluids 28 (1998) 129

15. Passoni, G., Alfonsi, G., Tula, G., Cardu, U.: A wavenumber parallel computational code for the numerical integration of the Navier-Stokes equations. Parall. Comp. 25 (1999) 593

16. Passoni, G., Cremonesi, P., Alfonsi, G.: Analysis and implementation of a parallelization strategy on a Navier-Stokes solver for shear flow simulations. Parall. Comp. 27 (2001) 1665

17. Passoni, G., Alfonsi, G., Galbiati, M.: Analysis of hybrid algorithms for the Navier-Stokes equations with respect to hydrodynamic stability theory. Int. J. Num. Meth. Fluids 38 (2002) 1069

18. Moser, R.D., Kim, J., Mansour, N.N.: Direct numerical simulation of turbulent channel flow up to $R e_{\tau}=590$. Phys. Fluids 11 (1999) 943 